\title{
Partial protection against enterovirus 71 (EV71) infection in a mouse model immunized with recombinant newcastle disease virus capsids displaying the EV71 VP1 fragment.
}

\begin{abstract}
Enterovirus 71 (EV71) infection may cause severe neurological complications, particularly in young children. Despite the risks, there are still no commercially available EV71 vaccines. Hence, a candidate vaccine construct, containing recombinant Newcastle disease virus capsids that display an EV71 VP1 fragment (NPt-VP1 1-100) protein, was evaluated in a mouse model of EV71 infection. Previously, it was shown that this protein construct provoked a strong immune response in vaccinated adult rabbits. That study, however, did not address the issue of its effectiveness against EV71 infection in young animals. In the present study, EV71 viral challenge in vaccinated newborn mice resulted in more than $40 \%$ increase in survival rate. Significantly, half of the surviving mice fully recovered from their paralysis. Histological analysis of all of the surviving mice revealed a complete clearance of EV71 viral antigens from their brains and spinal cords. In hind limb muscles, the amounts of the antigens detected correlated with the degrees of tissue damage and paralysis. Findings from this study provide evidence that immunization with the NPt-VP1 1-100 immunogen in a newborn mouse model confers partial protection against EV71 infection, and also highlights the importance of NPt-VP1 1-100 as a possible candidate vaccine for protection against EV71 infections.
\end{abstract}

Keyword: Enterovirus 71; Infection; Vaccine; Protection. 